\title{
Testing for CYP2C9 Before Anticoagulant Therapy
}

\author{
Mark H. Eckman, $M D, M S^{7}$, Steven M. Greenberg, $M D, P h D^{2}$, and Jonathan Rosand, $M D, M S c^{3}$ \\ 1. University of Cincinnati Medical Center, Cincinnati, OH, USA; ${ }^{2}$ Research Group, Department of Neurology, Massachusetts General Hospital, \\ Boston, MA, USA; ${ }^{3}$ Center for Human Genetic Research, Massachusetts General Hospital, Boston, MA, USA.
}

A uthors Reply: We share Dr. Jolobe's sentiment that appropriate tools must be developed to better characterize the risk of bleeding, particularly intracerebral hemorrhage, for patients with indications for oral anticoagulant therapy. Indeed, the increased use of warfarin anticoagulation for prevention of thromboembolic stroke in patients with atrial fibrillation (AF) has produced significant benefits, but has also resulted in an estimated quintupling of the incidence of warfarin-associated intracerebral hemorrhage (ICH). ${ }^{1}$ Warfarin-associated ICH now comprises roughly $20 \%$ of all $\mathrm{ICH}$. Furthermore, among patients with $\mathrm{ICH}$, warfarin is associated with a doubling in the case fatality rate at 3 months and an increase in poor neurological outcomes. ${ }^{2}$ There is suggestive evidence that the risk-adjusted incidence of cardioembolic ischemic stroke in patients with atrial fibrillation has declined over the past 2 decades, perhaps in response to more aggressive treatment of underlying risk factors such as hypertension and hyperlipidemia. ${ }^{3-5}$ As a result, the balance between the risk and benefit of anticoagulation therapy in patients with non-valvular atrial fibrillation may be shifting. These two trends, the increasing incidence of warfarin-associated $\mathrm{ICH}$ and the decreasing risk of cardioembolic ischemic stroke in patients with $\mathrm{AF}$, underscore the imperative to develop better tools to predict the risk of warfarin-associated ICH to improve "personalized" decision-making about anticoagulant therapy for patients with $\mathrm{AF}$.

Dr. Jolobe mentioned several possible risk markers for warfarin-associated $\mathrm{ICH}$, including genetic risk factors such as apolipoprotein $\mathrm{E}$ and imaging studies such as MRI and CT scanning. Indeed, in a previous publication we explored whether screening strategies that include genetic profiling and neuroimaging might improve the safety of chronic anticoagulation for atrial fibrillation by identifying individuals from whom warfarin should be withheld or for whom aspirin might be a better treatment. ${ }^{6}$ Unfortunately, we found that currently identified genetic markers of bleeding risk, such as Apo $€ 2$ or 4 do not confer a risk of ICH sufficiently high to warrant routine genetic testing for patients at average risk of thromboembolism. We did find that for patients at lower risk for cardioem-

Published online June 11, 2009 bolic stroke, screening with gradient echo MRI would be beneficial if the risk conferred by neuroimaging evidence of cerebral microhemorrhage were greater than 3.2-fold, a number somewhat higher than current estimates. ${ }^{7}$ Although studies will require large numbers of patients, given the infrequency of $\mathrm{ICH}$, it is imperative that trials be performed to better characterize the risk of future ICH associated with findings such as cerebral microhemorrhage or possibly novel genetic markers we have yet to identify.

Corresponding Author: Mark H. Eckman, MD, MS, , University of Cincinnati Medical Center, P.O. Box 670535, Cincinnati, OH 452670535, USA (e-mail: mark.eckman@uc.edu).

\section{REFERENCES}

1. Flaherty ML, Kissela B, Woo D, et al. The increasing incidence of anticoagulant-associated intracerebral hemorrhage. Neurology. 2007;68 (2):116-21

2. Rosand J, Eckman MH, Knudsen KA, Singer DE, Greenberg SM. The effect of warfarin and intensity of anticoagulation on outcome of intracerebral hemorrhage. Arch Intern Med. 2004;164(8):880-4.

3. Go AS, Hylek EM, Chang Y, et al. Anticoagulation therapy for stroke prevention in atrial fibrillation: how well do randomized trials translate into clinical practice? Jama. 2003;290(20):2685-92.

4. Singer DE, Albers GW, Dalen JE, et al. Antithrombotic therapy in atrial fibrillation: american college of chest physicians evidence-based clinical practice guidelines (8th Edition). Chest. 2008;133(6 Suppl):546S-592S.

5. Lip GY, Frison L, Grind M. Effect of hypertension on anticoagulated patients with atrial fibrillation. Eur Heart J. 2007;28(6):752-9.

6. Eckman MH, Wong LK, Soo YO, et al. Patient-specific decision-making for warfarin therapy in nonvalvular atrial fibrillation: how will screening with genetics and imaging help? Stroke. 2008;39(12):3308-15.

7. Soo YO, Yang SR, Lam Ww, et al. Risk vs benefit of anti-thrombotic therapy in ischaemic stroke patients with cerebral microbleeds. J Neurol. 2008;255(11):1679-86.

J Gen Intern Med 24(8):993

DOI: $10.1007 / \mathrm{s} 11606-009-1040-7$

(C) Society of General Internal Medicine 2009 